\title{
MATCHING ASYMPTOTIC METHOD IN PROPAGATION OF CRACKS WITH DUGDALE MODEL
}

\author{
Dang Thi Bach Tuyet ${ }^{1,}$, Jean Jacques Marigo ${ }^{1,}$ b and Laurence Halpern ${ }^{2,}$ c \\ ${ }^{1}$ Laboratoire de Mécanique des Solides (UMR 7649), École Polytechnique, 91128 Palaiseau cedex, \\ France \\ ${ }^{2}$ LAGA, Institut Galile, Universit Paris XIII, 93430 Villetaneuse, France \\ a tuyet@1ms.polytechnique.fr, ${ }^{\mathrm{b}}$ marigo@1ms.polytechnique.fr, ${ }^{\mathrm{c}}$ halpern@math.univ-paris13.fr
}

\begin{abstract}
Abstract. The goal of this work is to apply the matching asymptotic method combined with a variational approach to study the initiation and the propagation of a cohesive crack from the tip of a preexisting notch following the Dugdale cohesive force model when the characteristic length of the material (included in the Dugdale model) is small by comparison with the characteristic length of the body.
\end{abstract}

Key Words: Brittle fracture, matching asymptotic, anti-plane case, Dugdale model.

\section{INTRODUCTION}

Dugale model is based on the assumption that the surface energy density $\phi$ depends on the displacement jump, such as in the mode III, the displacement field at equilibrium $\mathbf{u}$ is antiplane, i.e.

$$
\mathbf{u}(\mathbf{x})=u\left(x_{1}, x_{2}\right) \mathbf{e}_{3}
$$

the surface energy density is formulated :

$$
\phi(\llbracket u \rrbracket)= \begin{cases}G_{c} \llbracket u \rrbracket / \ell_{c} & \text { if } \llbracket u \rrbracket \leq \delta_{c} \\ G_{c} & \text { if } \llbracket u \rrbracket \geq \delta_{c}\end{cases}
$$

In Eq.1, $G_{c}$ denotes for the critical energy release rate of the Griffith theory, whereas $\delta_{c}$ is an internal length characteristic of the cohesive forces model. $\llbracket u \rrbracket$ denotes for the jump of the displacement. The so-called cohesive foces given by the ratio $G_{c} / \delta_{c}$ is denoted $\sigma_{c}$ with $\sigma_{c}=\frac{G_{c}}{\delta_{c}}$. From Eq.1, the normal stress, such as $\sigma_{32}$, on the crack lips is equal $\sigma_{c}$ if $\llbracket u \rrbracket<\delta_{c}$ and eliminates if $\llbracket u \rrbracket>\delta_{c}$. Thus, the crack lips are divided into two zone : a cohesive zone where the cohesive forces are equal to $\sigma_{c}$ and a non cohesive zone where $\llbracket u \rrbracket>\delta_{c}$ where having non cohesive forces. Here, we are interested in studying the progress of initiation as well as propagation of a crack in the geometry of the notch characterized by the parameter $\epsilon=\tan \left(\pi-\frac{\omega}{2}\right), \omega$ being the angle of the notch, see Fig. 1. We will consider two scale of coordinates: the "macroscopic" coordinates $\mathbf{x}=\left(x_{1}, x_{2}\right)$ and the "microscopic" coordinates $\mathbf{y}=\mathbf{x} / \ell=\left(y_{1}, y_{2}\right)$, where $\ell$ is a small characteristic length of the crack inside the body. The tip of the notch is taken as the origin of the space. With supposing that crack appears inside the body follows a straight-path which derives from the notch tip and the axis $x_{1}$ is chosen in the same direction of crack propagation. The unit vector orthogonal to the $\left(x_{1}, x_{2}\right)$ plane is denoted $\mathbf{e}_{3}$.

\section{THE REAL PROBLEM}

The natural reference configuration of the sound two-dimensional body is denoted by $\Omega_{0}$. Assuming that a crack denoted by $\Gamma$ appears inside $\Omega_{0}$. The cohesive zone is denoted by $\Gamma_{c}$ and the non-cohesive zone is denoted by $\Gamma_{0}$. They are governed by their lengths of $\ell$ and $h: \Gamma=\Gamma_{0} \cup \Gamma_{c}$ with $\Gamma_{0}=(0, l) \times$ $\{0\} \quad$ and $\Gamma_{c}=[l, \ell) \times\{0\}$. The associated body containing the crack $\Gamma$ is denoted by $\Omega_{\Gamma}=\Omega_{0} \backslash \Gamma$. The two edges of the notch are denoted by $\Gamma^{+}$and $\Gamma^{-}$in Fig. 1. When one uses polar coordinates $(r, \theta)$, the pole is the tip of the notch and the origin of the polar angle is the edge $\Gamma^{-}$. Accordingly, we have 


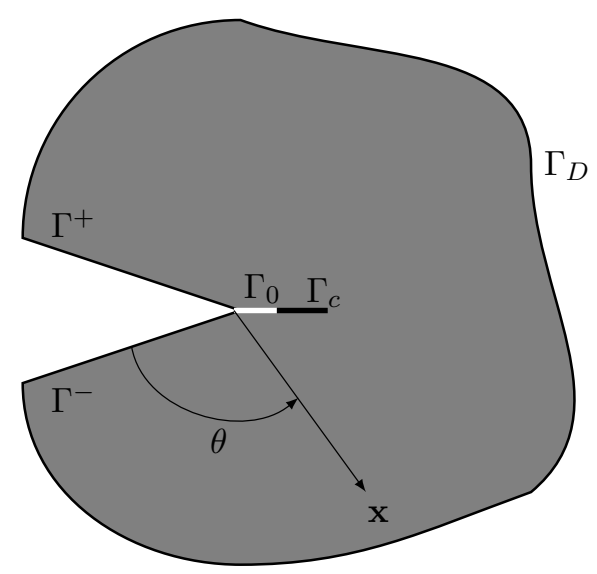

FIGURE 1. The domain $\Omega_{\Gamma}$ for the real problem

$: r=|\mathbf{x}|, \Gamma^{-}=\left\{(r, \theta), 0<r<r^{*}, \theta=0\right\}, \Gamma^{+}=\left\{(r, \theta), 0<r<r^{*}, \theta=\omega\right\}$. With assuming without body forces, $u$ must be an harmonic function : $\Delta u=0$ in $\Omega_{\Gamma}$

The edges of the notch are free while the lips of the crack are submitted to a cohesive forces :

$$
\frac{\partial u}{\partial \nu}=0 \quad \text { on } \quad \Gamma^{ \pm} \cup \Gamma^{0}, \quad \frac{\partial u}{\partial \nu}=\sigma_{c} \nu \quad \text { on } \quad \Gamma_{c}
$$

In (2), $\nu$ denotes the unit outer normal vector to the domain. The remaining part of the boundary of $\Omega_{\Gamma}$ is denoted by $\Gamma_{D}$ where the anti-plane displacement is prescribed such that the load is beyond the fracture threshold requires us to consider the initiation and the propagation of a non-cohesive part of the lips of the crack. Specifically, we have :

$$
u=U_{\infty} \quad \text { on } \quad \Gamma_{D}
$$

The linearity allows us to decompose the original problem into two problems corresponding to the value of $U_{\infty}$ on the boundary and $\sigma_{c}$ on the crack lips independently. The two problems will be denoted by the non-cohesive problem and the cohesive problem. We denote $u^{\infty}$ be the solution of the non-cohesive problem and $u^{c}$ be the solution of the cohesive problem.

The non-cohesive problem. Finding the solution $u^{\infty}$ such that it satisfies the set of the following equations:

$$
\left\{\begin{array}{lll}
\Delta u^{\infty}=0 & \text { in } & \Omega_{\Gamma} \\
\frac{\partial u^{\infty}}{\partial \nu}=0 & \text { on } & \Gamma_{N}^{+} \cup \Gamma_{N}^{-} \cup \Gamma_{0} \cup \Gamma_{c} \\
u^{\infty}=1 & \text { on } & \Gamma_{D}
\end{array}\right.
$$

The cohesive problem. Finding the solution $u^{c}$ such that it satisfies the set of the following equations:

$$
\begin{cases}\Delta u^{c}=0 & \text { in } \quad \Omega_{\Gamma} \\ \frac{\partial u^{c}}{\partial \nu}=0 & \text { on } \Gamma_{N}^{+} \cup \Gamma_{N}^{-} \cup \Gamma_{0} \\ \mu \nabla u^{c}=-\mathbf{e}_{2} & \text { on } \Gamma_{c} \\ u^{c}=0 & \text { on } \Gamma_{D}\end{cases}
$$

The displacement of the real problem is found through the solution $u^{\infty}$ of Eq. 4 and the solution $u^{c}$ of Eq. 5. It can be read as :

$$
u(\mathbf{x})=U_{\infty} u^{\infty}(\mathbf{x})-\sigma_{c} u^{c}(\mathbf{x})
$$

When the length $\ell$ is small comparision with the characteristic length of the body, MA method is applied because of the existing the two overlapped singularities near the notch tip and the crack tip. 


\section{MATCHING ASYMPTOTIC APPROACH}

The solution is found by two asymptotic expansions in terms of $\ell$. The first one, called the inner expansion, is valid in the neighborhood of the tip of the notch, ie. $r \ll 1$, while the other, called the outer expansion, is valid far from this tip, ie. $r \gg \ell$. These two expansions are matched in an intermediate zone. We approaching both of the problems Eq. 4 and Eq. 5 by using the MA expansion with respect to the asymptotic variable $\ell$ in order to obtain the more correct solution in vicinity of the crack. With Eq. 4, we have a homogeneous Neumann conditions on the crack lips, while in the Eq. 5 the Neuman conditions on the crack lips are constant.

MA approach for the non-cohesive problem. Supposing the outer expansion and the inner expansion of the solution $u^{\infty}$ is expanded in the series of $\ell$. We denote $u_{\text {out }}^{\infty}$ for the outer expansion of $u^{\infty}$ and the $u_{i n}^{\infty}$ for the inner expansion of $u^{\infty}$.

$$
u_{\text {out }}^{\infty}(\mathbf{x})=\sum_{i \in \mathbb{N}} \ell^{i \lambda} U^{i}(\mathbf{x}) \quad \text { and } \quad u_{i n}^{\infty}(\mathbf{x})=\sum_{i \in \mathbb{N}} \ell^{i \lambda} V^{i}(\mathbf{y})
$$

Each of the expanded terms such as $U^{i}$ and $V^{i}$ are determined by inserting Eq. 7 into Eq. 5. Even though the expansion of $u_{\text {out }}^{\infty}$ is valid far from the crack, $U^{i}$ must be defined in the whole outer domain $\Omega_{0}$ which corresponds to the sound body. However, the behavior of $U^{i}$ in the neighborhood of $r=0$ is singularity. Even though inner expansion valid only in the neighborhood of $r=0, V^{i}$ must be defined in the infinite inner domain $\Omega^{\infty}$. Moreover, the conditions at infinity is missing in the set of equations governed for $V^{i}$. The singularity at $r=0$ of $U^{i}$ and the conditions at infinity of $V^{i}$ will be given by the matching conditions.

MA approach for the cohesive problem. Being similar to the non-cohesive problem. We find the expanded forms of the outer expansion and inner expansion of the solution $u^{c}$. We denote $u_{\text {out }}^{c}$ for the outer expansion of $u^{c}$ and the $u_{i n}^{c}$ for the inner expansion of $u^{c}$. They can be read as :

$$
u_{\text {out }}^{c}(\mathbf{x})=\ell \sum_{i \in \mathbb{N}} \ell^{i \lambda} \bar{U}^{i}(\mathbf{x}) \quad \text { and } \quad u_{\text {in }}^{c}(\mathbf{x})=\ell \sum_{i \in \mathbb{N}} \ell^{i \lambda} \bar{V}^{i}(\mathbf{y})
$$

The process of finding the expanded terms,ie. $\bar{U}^{i}$ and $\bar{V}^{i}$ in Eq. 8 is similar to process for the noncohesive problem.

This work will be mentioned precisely in our article "Matching asymptotic method and nucleation of a defect of a notch" submitted in ACOME 2012. In the following section, we will introduce the criteria of minimum energy for determining the two tips of the cohesive zone and the non-cohesive zone.

\section{THE MINIMUM ENERGY APPROACHING}

At equilibrium sate, the total energy of the body can be read as :

$$
\mathcal{E}\left(U_{\infty}, l, \ell\right)=\frac{1}{2} \int_{\Omega_{\Gamma}} \nabla u . \nabla u d x-\int_{\partial \Omega} \nabla u \nu U^{\infty} d s+\int_{\Gamma_{c}} \sigma_{c} \llbracket u \rrbracket d x_{1}+2 G_{c}(\ell-l)
$$

where the last two terms which are considered as the surface energy of the body including the surface energy of the cohesive zone and non-cohesive zone. The evolution of the tip of non-cohesive zone and the tip of cohesive zone corresponding to the external load $U_{\infty}$ must be such that the total energy $\mathcal{E}\left(U_{\infty}, .,.\right)$ of the body obtain local minimum at $(l, \ell)$ for a given $U_{\infty}$. The minimum energy criteria can be read as :

$$
\frac{\partial \mathcal{E}}{\partial \ell}\left(U_{\infty}, l, \ell\right)=0 \quad \text { and } \quad \frac{\partial \mathcal{E}}{\partial l}\left(U_{\infty}, l, \ell\right)=0
$$

In other words, it proposes the tips of cohesive zone and non-cohesive zone are determined such that the total energy release rates due to the propagation of them in turn vanish. We denotes the energy release 
rates due to the propagation of the tip of the cohesive zone by $\mathcal{G}_{\ell}$ and the energy release rates due to the propagation of the tip of the non-cohesive zone by and $\mathcal{G}_{l}$. They can be formulated as following :

$$
\mathcal{G}_{\ell}=-\frac{\partial \mathcal{E}}{\partial \ell}\left(U_{\infty}, l, \ell\right), \quad \mathcal{G}_{l}(u)=-\frac{\partial \mathcal{E}}{\partial l}\left(U_{\infty}, l, \ell\right)
$$

Calculation of the energy release rate $\mathcal{G}_{\ell}$. Taking the first derivative in Eq. 11 meets some difficulties because of the existing the singularity at the tip of the cohesive zone. Following the idea of mapping the variable crack domain onto a fixed crack domain, we can compute $\mathcal{G}_{\ell}$. Supposing the crack in small, we use a smooth map to transform the neighborhood of the crack at the state of $\Gamma_{\bar{\ell}}(\bar{\ell}=\ell+\delta \ell)$ into the neighborhood of the crack at the state of $\Gamma_{\ell}$. Let $\mathbf{v}(\mathbf{y})=v(\mathbf{y}) \mathbf{e}_{1}$ be a smooth vector where $v(\mathbf{y})$ is defined :

$$
v(\mathbf{y})=\left\{\begin{array}{lll}
-\sqrt{\left(y_{1}-1\right)^{2}+y_{2}^{2}}+1 & \text { if } & \sqrt{\left(y_{1}-1\right)^{2}+y_{2}^{2}} \leq 1 \\
0 & \text { if } & \sqrt{\left(y_{1}-1\right)^{2}+y_{2}^{2}}>1
\end{array}\right.
$$

applying the MAE into the formulating of $\mathcal{G}_{\ell}$, using the inner expansion, finally, we obtain :

$$
\mathcal{G}_{\ell}=\frac{\mu}{\ell} \int_{B(1,1) \backslash \Gamma_{1}}\left(\frac{\partial u^{i n}}{\partial y_{j}} \frac{\partial u^{i n}}{\partial y_{1}} \frac{\partial v}{\partial y_{j}}-\frac{1}{2} \frac{\partial u^{i n}}{\partial y_{j}} \frac{\partial u^{i n}}{\partial y_{j}} \frac{\partial v}{\partial y_{1}}\right) d s-\int_{\Gamma_{c}} \sigma_{c} \llbracket u^{i n} \rrbracket \frac{\partial v}{\partial y_{1}} d y_{1}
$$

where $B(1,1)$ is notation for a zone defined by $\left\{\left(y_{1}, y_{2}\right) \in \Omega^{\infty}\right.$ such that $\left.\left(y_{1}-1\right)^{2}+y_{2}^{2} \leq 1\right\}$. Because of Eq. 6, accordingly, the stress field $\sigma$ and the SIF $K_{I I I}$ can be expressed as :

$$
\sigma(\mathbf{x})=U_{\infty} \sigma^{\infty}(\mathbf{x})-\sigma_{c} u^{c}(\mathbf{x}) \quad \text { and } \quad K_{I I I}=U_{\infty} K_{I I I}^{\infty}-\sigma_{c} K_{I I I}^{c}
$$

where $\left(\sigma^{\infty}(\mathbf{x}), K_{I I I}^{\infty}\right)$ and $\left(\sigma^{c}(\mathbf{x}), K_{I I I}^{c}\right)$ are the stress and the SIF at the tip of the cohesive zone coressponding to $u^{\infty}$ and $u^{c}$. Besides, because the cohesive forces are constant, they do not change the form of the singularity at the tip of the crack of the cohesive zone. The criteria governing the propagation of the cohesive crack tip $\mathcal{G}_{\ell}$ is equivalent to SIF $K_{I I I}=0$. Irwin formula give us that $\mathcal{G}_{\ell} \sim K_{I I I}^{2}$, moreover, Eq. 14 leads to :

$$
U_{\infty}=\sigma_{c} \sqrt{\mathcal{G}_{\ell}^{c} / \mathcal{G}_{\ell}^{\infty}}
$$

with $\mathcal{G}_{\ell}^{c}$ and $\mathcal{G}_{\ell}^{\infty}$ are defined as in Eq. 13 but $u^{i n}$ is replaced by corresponding $u^{\infty}$ and $u^{c}$.

Calculation of the energy release rate $\mathcal{G}_{l}$. There is no singularity at the tip of non-cohesive zone, so $\mathcal{G}_{l}$ can obtained by taking the derivative of $\mathcal{E}\left(U_{\infty}, l, \ell\right)$ as in Eq. 9 with respect to $l$ under the integration. It can be read as :

$$
\mathcal{G}_{l}=2 \sigma_{c} \llbracket u \rrbracket(l)-2 G_{c}
$$

besides, Eq. 10 and Eq. 11 lead to :

$$
\llbracket u \rrbracket(l)=G_{c} / \sigma_{c} \quad \text { or } \quad U_{\infty} \llbracket u \rrbracket^{\infty}(l)-\sigma_{c} \llbracket u \rrbracket^{c}(l)=\frac{G_{c}}{\sigma_{c}}
$$

Accordingly, we obtain the system of two coupled non linear equations of Eq. 15 and Eq. 17 which determine the tips of the cohesive zone and the non-cohesive zone.

Acknowledgements. This work was partially supported by the French Agence Nationale de la Recherche (ANR), under grant epsilon (BLAN08-2_312370) "Domain decomposition and multi-scale computations of singularities in mechanical structures". 


\section{REFERENCES}

[1] B. Bourdin, G. A. Francfort, and J.-J. Marigo. The variational approach to fracture. Springer, 2008.

[2] M. Dauge, S. Tordeux, and G. Vial. Selfsimilar perturbation near a corner: matching versus multiscale expansions for a model problem. In Around the research of Vladimir Maz'ya. II, volume 12 of Int. Math. Ser. (N. Y.), pages 95-134. Springer, 2010.

[3] P. Grisvard. Elliptic problems in non smooth domains. Number 24 in Monographs and Studies in Mathematics. Pitman, 1985.

[4] J.-B. Leblond. Mécanique de la rupture fragile et ductile. Collection Études en mécanique des matériaux et des structures. Editions Lavoisier, Paris, 2000.

[5] J.-J. Marigo. Initiation of cracks in Griffith's theory: an argument of continuity in favor of global minimization. Journal of Nonlinear Science, 20(6):831-868, 2010. 